PAPER

\title{
The features of myasthenia gravis with autoantibodies to MuSK
}

\author{
D Lavrnic, M Losen, A Vujic, M De Baets, L J Hajdukovic, V Stojanovic, R Trikic, P Djukic, \\ S Apostolski
}

J Neurol Neurosurg Psychiatry 2005;76:1099-1 102. doi: 10.1136/jnnp.2004.052415

\begin{abstract}
See end of article for authors' affiliations

.....................

Correspondence to: Dr D Lavrnic, Institute of Neurology, Clinical Centre of Serbia, 11000 Belgrade, 6 Dr Subotica Street, Serbia and Montenegro; joltruth@ sezampro.yu
\end{abstract}

Received 22 August 2004 In revised form

8 November 2004

Accepted

16 December 2004 if myasthenia gravis (MG) with antibodies to MUSK is a distinct subgroup of Objectives: To determine if myasthenia gravis (MG) with antibodies to MUSK is a distinct subgroup of
seronegative MG. Methods: We assayed antibodies to muscle specific tyrosine kinase (MUSK) in 55 MG patients who had no antibodies to acetylcholine receptors and looked for the specific phenotype, comparing clinical features of anti-MuSK positive and anti-MuSK negative MG patients.

Results: MG with anti-MuSK antibodies was characterised by a striking prevalence of female patients (15 women, two men). Age at onset ranged from 22 to 52 years, with $70.6 \%$ of patients presenting at $<40$ years of age. The majority of patients $(82.4 \%)$ had prevalent involvement of facial and bulbar muscles. One third of them did not respond well to anticholinesterase drugs. Steroid immunosuppression was effective in eight patients $(44.4 \%)$. Nine patients underwent thymectomy; six of these had no thymus pathology, while three had a hyperplastic thymus. At the end of the observation period, six (35.3\%) patients were in remission, five (29.4\%) improved, four (23.6\%) did not change, and two (11.7\%) had died.

Conclusions: MG patients with antibodies to MUSK have characteristic clinical features that are different from features of the remaining seronegative MG patients. This emphasises the predictive value of antiMuSK antibody analysis in seronegative MG patients.
M yasthenia gravis (MG) is an autoimmune disorder of the skeletal muscles characterised by a decrease in the number of available acetylcholine receptors (AChR). Approximately $80-90 \%$ of patients have detectable serum anti-AChR antibodies, ${ }^{1}$ but there is conclusive evidence that patients without these antibodies also have an antibody mediated disorder. ${ }^{2}$ Recently, it was shown that approximately $40-50 \%$ of seronegative MG (SNMG) patients have antibodies to a surface membrane enzyme, muscle specific tyrosine kinase (MUSK), which is responsible for agrin induced AChR clustering at the postsynaptic membrane. ${ }^{3}$ A specific clinical phenotype of SNMG has been described in patients with anti-MuSK antibodies, ${ }^{4}$ but these antibodies have been also detected in seropositive MG with or without thymoma. ${ }^{5}$ We analysed the clinical, electrophysiological, pharmacological, and pathological features in 17 SNMG patients with anti-MuSK antibodies.

\section{METHODS}

More than 1200 patients with MG have been treated at the Institute of Neurology, Clinical Centre of Serbia, in Belgrade. In all, the diagnosis of MG was based on a typical clinical pattern, a positive neostigmine and/or edrophonium test and decreasing response on repetitive nerve stimulation (performed on the facial nerve-nasal muscle and axillary nervedeltoid muscle systems) and/or increased jitter in single fibre EMG. During the past 2 years anti-AChR antibodies were analysed in the sera of 276 patients: $221(80.1 \%)$ patients were positive and 55 (19.9\%) were negative. Recently, we assayed anti-MuSK antibodies in all seronegative MG patients; 40 women and 15 men. At the time these patients were diagnosed and treated we were unable to perform either anti-AChR Ab or anti-MuSK Ab analyses, thus we did not know the patients' antibody status. Consequently, all the patients were treated according to the clinical form of the disease, regardless of the presence of antibodies.
The severity of MG was examined at the peak of the disease and graded by using the modified Osserman's scale and the modified Besinger's standardised clinical score. ${ }^{6}$ Four degrees of muscle weakness were determined. Full muscle strength was graded 0 , fatigability 1 , moderate to severe weakness 2, and total loss of function 3. The severity of the disease was expressed as the mean disability score (MDS), which was obtained by adding the scores for each of the nine examined muscle groups (extraocular, jaw, facial, bulbar, neck, respiratory, proximal and distal upper limb muscles, and proximal lower limb muscles). In every patient, the MDS was determined at the peak of disease (MDS1) and at the end of the follow up period (MDS2). The outcome was expressed by the mean disability score quotient (MDSQ), which was obtained by MDSQ = MDS2/MDS1. An MDSQ between 0.5 and 1 indicated no improvement, while values between 0 and 0.5 were considered as improvement. Patient was considered to be in remission when MDSQ was 0. Pharmacological remission was defined as the absence of any myasthenic signs with the therapy, and complete remission the absence of any myasthenic signs without any therapy.

The clinical characteristics of anti-MuSK positive patients were compared with those of anti-MuSK negative SNMG patients. All patients have been followed longitudinally for 3-10 years.

The concentration of anti-AChR antibodies was measured by a standard radioimmunoassay method using AChR radioimmunoassay kits (CIS Biointernational). Anti-MuSK antibodies were detected using the MuSK Ab Assay kit (RSR Ltd, Cardiff, UK), according to the manufacturer's protocol. Briefly, $5 \mu \mathrm{L}$ of serum was incubated with $50 \mu \mathrm{L}$ of

Abbreviations: AChR, acetylcholine receptors; MDS, mean disability score; $M D S Q$, mean disability score quotient; $M G$, myasthenia gravis; MuSK, muscle specific tyrosine kinase; SNMG, seronegative myasthenia gravis 
${ }^{125}$ I-MuSK (extracellular domains) for 16 hours. Human antibodies were immunoprecipitated for 2 hours, centrifuged, and the washed pellets were measured in a gamma counter.

\section{Statistical analysis}

For descriptive presentation of numerical and attributive variables, absolute and relative frequencies were calculated. The numerical variables are presented as means (SD). The statistical evaluation of the data was performed using SPSS version 10.0 for Windows. A $\chi^{2}$ test with Yates's correction was used to compare the frequency distribution of different gradations of categorical variables in the group of patients with and without anti-MuSK antibodies, and also to compare the difference in the frequency distribution of the same variable between these two groups of patients. Values of $\mathrm{p}<0.05$ were considered to be statistically significant.

\section{RESULTS}

Among the 55 SNMG patients, 17 were anti-MuSK antibody positive (30.9\%) and 38 (69.1\%) were anti-MuSK Ab negative. In the group of patients with anti-MuSK antibodies there was a striking prevalence of females. Fifteen patients were women $(88.2 \%)$ and only two $(11.8 \%)$ were men (female:male ratio 7.5:1) ( $\chi^{2}$ with Yates's correction $=8.4$, d.f. $=1, p<0.01)$. Among anti-MuSK negative SNMG patients there was also a female preponderance ( 24 females $(63.2 \%)$ and 14 males (36.8\%); female:male ratio $1.7: 1)$, but without statistical significance $\left(\chi^{2}\right.$ with Yates's correction $=2.2$, d.f. $=1, \mathrm{p}>0.05)$. However, the difference in sex distribution between anti-MuSK positive and anti-MuSK negative patients was of no statistical significance $\left(\chi^{2}\right.$ with Yates's correction $=2.4$, d.f. $=1, \mathrm{p}>0.05)$. The age at onset of the anti-MuSK positive MG patients ranged from 22 to 52 years (mean (SD (35.6 (10.3) years); in 12 of 17 patients $(70.6 \%)$, onset of the disease was before the age of 40 years. The age at onset of the disease in anti-MuSK negative patients was similar: 38.3 (12.8) years (range 14 to 63 years).

In nine of 17 anti-MuSK positive MG patients (52.9\%), the first symptoms of the disease were oculobulbar, in three (17.6\%) bulbar, in two (11.8\%) ocular, and in the remaining three $(17.6 \%)$ generalised $\left(\chi^{2}=7.3\right.$, d.f. $\left.=3, p>0.05\right)$. At the peak of the disease, 14 anti-MuSK positive patients $(82.4 \%)$ had a severe generalised form of MG (IIB), and only three patients (17.6\%) had the mild generalised form (IIA) of the disease (fig 1). The majority of the patients shared a similar pattern of muscle weakness, with prevalent involvement of facial and bulbar muscles (in 14 of 17 patients; $82.4 \%$ ). Six patients $(35.3 \%)$ developed respiratory muscle weakness and underwent endotracheal intubation with artificial ventilation. In anti-MuSK negative SNMG patients, 11 patients (29\%) had the ocular form (I) at the peak of the disease, 17 $(44.7 \%)$ had the mild generalised form (IIA), and $10(26.3 \%)$ had the severe generalised form (IIB), according to Osserman's classification (fig 1). By contrast, anti-MuSK positive patients had more severe forms of the disease $\left(\chi^{2}=15.8\right.$, d.f. $\left.=2, \mathrm{p}<0.01\right)$. In the chronic phase of the disease, three anti-MuSK positive patients (17.6\%) developed severe atrophy of facial and lingual muscles, while the same feature was observed in only one anti-MuSK negative patient $(2.6 \%)$ ( $\chi^{2}$ with Yates's correction $=2.15$, d.f. $\left.=1, p>0.05\right)$. Despite the fact that all patients had generalised muscle weakness, a decreasing response on repetitive nerve stimulation was registered in only eight anti-MuSK positive patients $(47.1 \%$ ) (fig 2). Single fibre EMG was performed in 11 patients and was positive in all (100\%). In anti-MuSK negative SNMG patients, decreasing response on repetitive nerve stimulation was registered in 23 of 27 patients (85.2\%) with generalised forms of the disease (fig 2). The sensitivity

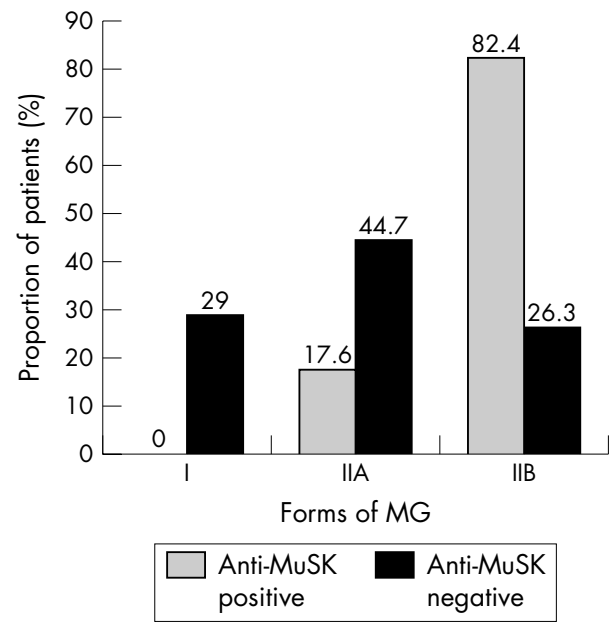

Figure 1 MG forms (according to Ossermans' classification) in SNMG patients. $p<0.01$.

of repetitive nerve stimulation was significantly lower in antiMuSK positive patients $\left(\chi^{2}\right.$ with Yates's correction $=5.6$, d.f. $=1, \mathrm{p}<0.05)$.

The effect of neostigmine or edrophonium was negative or equivocal in five anti-MuSK positive patients (29.4\%). Five patients $(29.4 \%)$ had hypersensitive response to usual single dose of pyridostigmine (60 mg) (fig 2). This hypersensitivity was manifested by severe muscle fasciculations, especially in the ocular and facial muscles, blurred vision, hypersalivation, and abdominal cramps. In anti-MuSK negative SNMG patients, hypersensitive response to pyridostigmine was observed in only one patient $(2.6 \%)\left(\chi^{2}\right.$ with Yates's correction $=6.0$, d.f. $=1, p<0.05$ ) (fig 2). Owing to hypersensitive response to pyridostigmine, all these patients were treated with very small, divided doses of 10-30 mg at every 4-8 hours. The remaining patients received pyridostigmine as doses of $60 \mathrm{mg}$ every $4-8$ hours. In addition to anticholinesterase drugs, all anti-MuSK positive and 35 antiMuSK negative (92\%) patients were also treated with corticosteroid drugs (average dose $60 \mathrm{mg}$ of prednisone on alternate day). In the patients who did not respond well to the treatment with anticholinesterases and corticosteroids, additional immunosuppressive drugs were added. Azathioprine was administered in five anti-MuSK positive

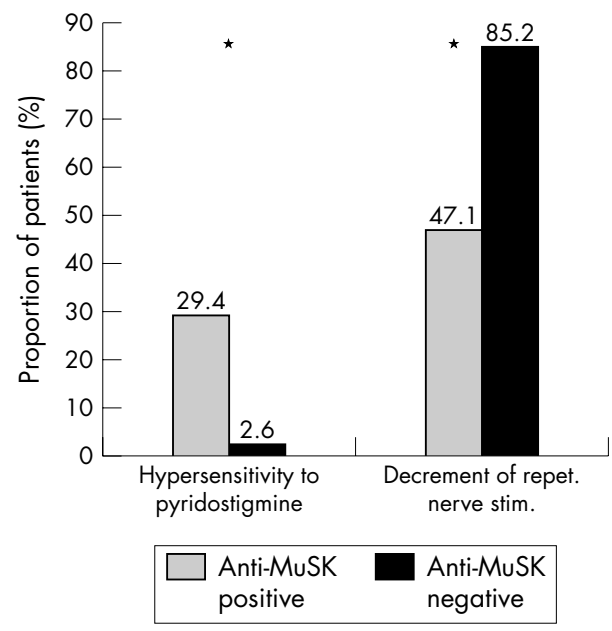

Figure 2 Hypersensitivity to pyridostigmine and decremental response on repetitive nerve stimulation in SNMG patients. ${ }^{*} p<0.05$. 
(29.4\%) and 14 anti-MuSK negative (36.8\%) patients. Three anti-MuSK positive $(17.6 \%)$ and one anti-MuSK negative $(2.6 \%)$ patient were treated with ciclosporin A, and one antiMUSK positive patient $(5.9 \%)$ was treated with mycophenolate mofetil. In the group of eight anti-MuSK positive patients successfully treated with corticosteroid drugs, two were in complete remission, four were in pharmacological remission, and two improved significantly. In the group of anti-MuSK positive patients treated with a combination of immunosuppressive drugs, two patients had to be treated additionally with intravenous immunoglobulin and four with plasma exchange because of an acute exacerbation and respiratory crisis. Among them, five patients improved but one died at 56 years of age, 8 years after the onset of the disease. Thymectomy was performed in nine of 17 (52.9\%) anti-MuSK positive MG patients. The criteria for thymectomy were the generalised form of the disease and age $<50$ years. None of the patients had a thymoma. A hyperplastic thymus was found in three patients $(17.6 \%)$, aged 23,32 , and 48 years, and a normal or atrophic thymus gland in the remaining six. In the group of anti-MuSK negative SNMG patients, thymectomy was performed in 17 of 27 patients with generalised forms of the disease $(63 \%)$. Thymoma was found in two (11.8\%), hyperplastic thymus in seven $(41.2 \%)$, and atrophic or persistent thymus gland in eight (47\%).

At the end of the follow up period, two anti-MuSK positive patients $(11.8 \%)$ were in complete remission, four $(23.5 \%)$ were in pharmacological remission, five (29.4\%) had improved, four $(23.5 \%)$ had an unchanged condition, and two patients had died $\left(\chi^{2}=2.2\right.$, d.f. $\left.=4, p>0.05\right)$ (fig 3). One patient died from a myasthenic crisis at the age of 56 years and the other, aged 24 years, from an acute intestinal necrosis. The latter patient was diagnosed 2.5 months prior to her death, and she received corticosteroids in dosage $20 \mathrm{mg}$ daily for 15 days and then $40 \mathrm{mg}$ daily. She was not thymectomised. She had associated thrombophilia, which was probably the cause of the lethal acute intestinal necrosis. Among the thymectomised patients, two achieved complete remission, two were in pharmacological remission, one significantly improved, three had an unchanged condition, and one died. Among the anti-MuSK negative SNMG patients, at the end of the follow up period, four (10.5\%) patients were in complete remission, five (13.2\%) in pharmacological remission, $20(52.6 \%)$ had improved, and nine $(23.7 \%)$ had an unchanged condition (fig 3$)$. There was no statistically significant difference in the outcome of the disease between the anti-MuSK positive and negative patients $\left(\chi^{2}=6.8\right.$, d.f. $\left.=4, \mathrm{p}>0.05\right)$.

We noticed associated disorders in nine (53\%) anti-MuSK positive MG patients: systemic lupus erythematosus (two

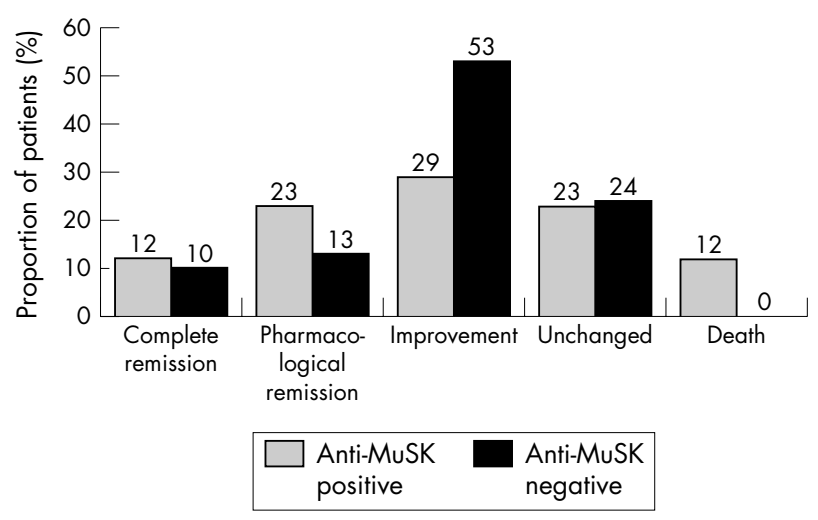

Figure 3 The outcome of MG in SNMG patients. $p>0.05$. patients), myoma uteri (three), allergy to penicillin (one), duodenal ulcer (one), carcinoma (one), struma nodosa (one), and thrombophilia (one). Among the SNMG anti-MuSK negative patients, associated diseases were found in seven (18.4\%) patients: hyperthyreosis (four patients), lung cancer (one), struma nodosa (one), bronchial asthma (one), and pulmonary fibrosis (one). The occurrence of associated diseases was significantly higher in the anti-MuSK positive patients $\left(\chi^{2}\right.$ with Yates's correction $=5.4$, d.f. $\left.=1, \mathrm{p}<0.05\right)$. In contrast, there was no statistically significant difference in the frequency of each disorder, either in the group of antiMuSK positive patients or between the anti-MuSK positive and anti-MuSK negative SNMG patients $(\mathrm{p}>0.05)$.

\section{DISCUSSION}

We detected anti-MuSK antibodies in 17 of 44 (38.6\%) our patients with generalised SNMG. The frequency is lower than the $40-70 \%$ frequency reported in other studies of white patients, $^{3}$ 4-9 $^{10}$ and significantly higher than the $4 \%$ reported in Chinese patients. ${ }^{11}$ The female preponderance was the significant feature of our anti-MuSK positive MG patients, which was in accordance with the finding in other studies of white patients, ${ }^{4810}$ but not with the study in Chinese patients, ${ }^{11}$ nor in the study published by Zhou et al. ${ }^{12}$ In $70 \%$ of our patients, the onset of the disease was before the age of 40 years (young onset MuSK-MG). Similar results (57\%) were published by Evoli et al. ${ }^{4}$ We found predominant involvement of facial and bulbar muscles in $82.4 \%$ of patients, similar to papers reporting prevalent oculobulbar weakness in anti-MuSK positive MG patients. ${ }^{37910}$ The weakness of masticatory, facial, and lingual muscles characterised the majority of our patients, and in the chronic phase of the disease, some of these patients (17.6\%) developed severe atrophy of these muscles. A high frequency of respiratory crisis $(35.3 \%)$ was similar to observations reported by other groups. ${ }^{3}$ The diagnostic value of neostigmine or edrophonium test and repetitive stimulation was low in our patients. Incomplete response to anticholinesterase drugs has been reported by several groups, ${ }^{378}$ but we observed hypersensitive reaction to average doses of oral pyridostigmine with exacerbation of muscle weakness and cholinergic signs in almost $30 \%$ of patients. Almost half of the patients $(47 \%)$ responded to corticosteroid immunosuppression, while the others needed to be treated with a combination of immunosuppressive drugs and with an intermittent plasma exchange or intravenous immunoglobulin. Most papers reported no benefit from thymectomy in anti-MuSK positive MG patients, ${ }^{48}$ but we observed significant improvement or remission in more than a half of thymectomised patients. We also noticed better response to corticosteroid treatment in thymectomised patients. The majority had no thymus pathology, but three of nine thymectomised patients had a hyperplastic thymus. In spite of the fact that our patients with anti-MuSK antibodies and MG have specific clinical, electrophysiological, pharmacological, and pathological features, we still do not know if these antibodies are the primary cause of the disease. ${ }^{13}$

\section{CONCLUSIONS}

Our results indicate that MG with antibodies to MuSK has a different phenotype from the remaining seronegative MG. MG with anti-MuSK antibodies was characterised by a striking prevalence of females (7.5:1), severe, predominantly faciobulbar weakness (82.4\%), and more frequent occurrence of facial and lingual muscle atrophy (17.6\%). Anti-MuSK MG patients frequently develop hypersensitivity to anticholinesterase drugs $(29.4 \%)$. Thymus pathology is less common in this subgroup of patients, but thymectomy is still beneficial. Routine repetitive nerve stimulation test has a 
low, while single fibre EMG has very high sensitivity. The specific clinical, electrophysiological, and immunological presentation, the thymus pathology, and the therapeutic response, particularly to anticholinesterase drugs, implicate that MG with anti-MuSK antibodies is a specific subgroup of seronegative MG. This emphasises the predictive value of anti-MuSK antibody analysis in all seronegative MG patients.

\section{Authors' affiliations}

D Lavrnic, A Vujic, V Stojanovic, R Trikic, S Apostolski, The Institute of Neurology, Clinical Centre of Serbia, Belgrade, Serbia and Montenegro P Diukic, Institute of Cardiovascular Surgery, Clinical Centre of Serbia, Belgrade, Serbia and Montenegro

M Losen, M De Baets, Department of Cellular Neuroscience, Universiteit Maastricht, The Netherlands

L J Hajdukovic, Institute for the Application of Nuclear Energy, Belgrade, Serbia and Montenegro

Competing interests: none declared

\section{REFERENCES}

1 Lindstrom JM. Acetylcholine receptors and myasthenia gravis. Muscle Nerve 2000;23:453-77.

2 Burges J, Vincent A, Molenaar PC, et al. Passive transfer of seronegative myasthenia gravis to mice. Muscle Nerve 1994;17:1393-400.
3 Hoch W, McConville J, Helms S, et al. Autoantibodies to the receptor tyrosine kinase MuSK in patients with myasthenia gravis without acetylcholine receptor antibodies. Nat Med 2001;7:365-8.

4 Evoli A, Tonali PA, Padua L, et al. Clinical correlates with anti-MuSK antibodies in generalized seronegative myasthenia gravis. Brain 2003; 126:2304-11

5 Ohta K, Shigemoto K, Kubo S, et al. MuSK antibodies in AChR Ab-seropositive MG vs AChR Ab-seronegative MG. Neurology 2004;62:2132-3.

6 Besinger UA, Toyka KV, Homberg M, et al. Myasthenia gravis: long term correlation of binding and bungarotoxin blocking antibodies against acetylcholine receptors with changes in disease severity. Neurology 1983;33:1316-21.

7 Vincent A, Bowen J, Newsom-Davis J, et al. Seronegative generalized myasthenia gravis: clinical features, antibodies, and their targets. Lancet Neurol 2003;2:99-106.

8 Sanders DB, El-Salem K, Massey JM, et al. Clinical aspects of MuSK antibody positive seronegative MG. Neurology 2003;60:1978-80.

9 Scuderi F, Marino M, Colonna L, et al. Anti-p1 10 autoantibodies identify a subtype of "seronegative" myasthenia gravis with prominent oculobulbar involvement. Lab Invest 2002;82:1139-46.

10 McConville J, Farrugia ME, Beeson D, et al. Detection and characterization of MuSK antibodies in seronegative myasthenia gravis. Ann Neurol 2004;55:580-4.

11 Yeh J-H, Chen W-H, Chiu H-C, et al. Low frequency of MuSK antibody in generalized seronegative myasthenia gravis among Chinese. Neurology 2004;62:2131.

12 Zhou L, McConville J, Chaudhry V, et al. Clinical comparison of musclespecific tyrosine kinase (MUSK) antibody-positive and -negative myasthenic patients. Muscle Nerve 2004;30:55-60.

13 Selcen D, Fukuda T, Shen X-M, et al. Are MuSK antibodies the primary cause of myasthenic symptoms? Neurology 2004;62:1945-50. 